\title{
DIVISION VI / COMMISSION 34 / WORKING GROUP ASTROCHEMISTRY
}

\author{
CHAIR \\ VICE-CHAIR \\ BOARD
}

\author{
E.F. van Dishoeck \\ E. Herbst \\ Y. Aikawa \\ J.H. Black \\ G.A. Blake \\ P. Caselli \\ J. Cernicharo \\ G. Garay \\ M. Guelin \\ U.G. Jørgensen \\ J.P. Maier \\ K.M. Menten \\ T.J. Millar \\ S. Kwok \\ F. Salama \\ I. Sims
}

A. Sternberg

\section{TRIENNIAL REPORT 2009-2012}

\section{Introduction}

The study of molecules in space, known as astrochemistry or molecular astrophysics, is a rapidly growing field. Molecules exist in a wide range of environments in both gaseous and solid form, from our own solar system to the distant early universe. To astronomers, molecules are indispensable and unique probes of the physical conditions and dynamics of regions in which they are detected, especially the interstellar medium. In particular, the many stages of both low-mass and high-mass star formation are better understood today thanks to the analysis of molecular observations. Molecules can also yield a global picture of the past and present of sources. Moreover, molecules affect their environment by contributing to the heating and cooling processes that occur.

Molecular observations are currently being used to study the interstellar gas and dust in diffuse interstellar clouds, dense molecular clouds, protostellar objects, maser and star-forming regions, envelopes of evolved stars, protoplanetary disks, (exo-)planetary atmospheres and comets. In addition to our own Milky Way Galaxy, molecules and solidstate features are routinely detected in interstellar regions of external galaxies ranging from the nearby Magellanic Clouds to distant starbust galaxies at redshifts of more than 6 . Indeed, the role of molecules in the study of the early universe, including the formation of the first stars, is a critical one. Molecules are also key probes of galactic assembly processes, e.g., mergers vs. smooth accretion, and molecular gas provides the fuel for star formation and black hole activity. The role of molecules in astronomy has 
grown to such an extent that it is no longer an exaggeration to refer to a sizable portion of the universe as 'The Molecular Universe'.

To chemists, the synthesis of molecules from simple atoms to complex species is of increasing interest. The distant goal of learning about the onset of pre-biotic processes is coming closer to home through studies of cometary samples and meteorites, as well as of circumstellar disks, which are the precursors of planets. The processes that produce these species are unusual and exciting since interstellar space provides a laboratory with conditions not readily simulated on Earth. Thus, astrochemistry attracts scientists from a number of different disciplines. Astrochemistry directly connects with even newer fields such as astrobiology and the study of exoplanets and their atmospheres. As a result, astrochemistry has many adherents throughout the world, and the potential for huge growth as new and more powerful telescopes search the sky for molecules. Large-scale international cooperation occurs in the construction and exploitation of expensive telescopes, such as space-based Herschel and JWST, air-borne SOFIA, and ground-based ALMA, for which the initial scientific cases were largely driven by astrochemists.

The IAU working group (WG) on Astrochemistry in Division VI (new Division H), Commission 34 (Interstellar Matter) was established about 30 years ago by Alexander Dalgarno and led by him for the first two IAU Symposia. From 1991-1999, the WG was chaired by David Williams, with Ewine van Dishoeck as vice-chair. From 1999-now, the WG is chaired by Ewine van Dishoeck with Eric Herbst as vice-chair.

The current WG members come from 13 countries and represent different disciplines (see http://www.strw. leidenuniv.nl/iau34/for overview). The WG is responsible for fostering interest in the subject and ensuring the continuation of meetings on the subject under the auspices of the IAU. So far, six IAU-sponsored meetings have been held, roughly every 5-6 years: IAU Symposium 120, in Goa, India 1985 on 'Astrochemistry'; IAU Symposium 150 on 'Astrochemistry of Cosmic Phenomena' in Brazil 1991, attended by 120 scientists from 19 countries; IAU Symposium 178, 'Molecules in Astrophysics: Probes and Processes' in Leiden, The Netherlands 1996, with 231 participants from 27 countries; IAU Symposium 197 'Astrochemistry: From Molecular Clouds to Planetary Systems' in Sogwipo, Korea 1999, with 262 participants from 25 countries; IAU Symposium 231 'Astrochemistry: Recent Successes and Current Challenges' in Asilomar, USA in 2005 with 300 participants from 26 countries, and IAU Symposium 280 'The Molecular Universe' in Toledo in 2011 with 440 participants from 30 countries. Worldwide, the number of astrochemists is expected to be at least double this number. The activity and growth of the field is further illustrated by a number of other international meetings and workshops around the world, including numerous meetings having to do with planning for the Herschel and ALMA telescopes, as well as dedicated networks on astrochemistry on a regional and continental basis. The purpose of the IAU symposia has been to ensure that the many aspects of astrochemistry are reviewed, and that the entire community (both astronomers and chemists/physicists) is brought together.

\section{Developments within the past triennium}

During the 2009-2012 triennium, the WG concentrated on the proposal, planning and organization of IAU Symposium 280 on 'The Molecular Universe' in beautiful Toledo, Spain, which took place from 30 May - 3 June 2011 at the newly renovated Technological Campus of the University of Castilla-La Mancha. The IAU WG acted as the Scientific Organizing Committee of the symposium. The Local Organizing Committee, chaired by J. Cernicharo, organized both the scientific and structural aspects of the meeting very well, including a delightful banquet and preceding concert. The cultural mecca that is 
Toledo added a sense of awe and excitement to the symposium. The large size of the meeting did not interfere with the proceedings in any way. A large number of questions were asked of speakers, who, given their relative youth and diversity, brought many different viewpoints to the proceedings. The three dedicated 2.5-hr. poster sessions were very well attended and enriched the experience of the participants. The sessions were enlivened by tapas and by a variety of beverages. Informal conversations at intermissions and during the poster sessions were many and spirited. The large number of younger scientists at the meeting was quite impressive, and confirmed that the field of Astrochemistry is entering a period of rapid growth led by new and exceedingly powerful telescopes.

The scientific program of the symposium consisted of 41 invited and review talks, 32 contributed talks, and 323 poster presentations. The SOC democratically proposed and elected the speakers. In the oral program were three sessions on new results from the Herschel Space Observatory labeled 'Herschel hot results', as well as a panel discussion entitled 'On to ALMA'. Three awards were given to the best posters in each of the three sessions from personal funds by Ewine van Dishoeck, as well as a prize for the best proposal in the ALMA session. During the third poster session, there were also computer demonstrations of databases. The abstracts for all contributions to the symposium can be found on the NASA Astrophysics Data System and on the conference website: http://www.cab.inta-csic.es/molecular_universe/show-abstracts.php, where actual poster presentations have been uploaded. A number of video interviews and highlights of the poster sessions can be found on the IAU Symposium 280 YouTube Channel (http://www.youtube.com/user/IAUsymposium280). Invited and review talks will appear in the symposium volume, edited by J. Cernicharo and R. Bachiller.

The symposium started with a session on star formation. This field has become broader since the last astrochemistry symposium, and observational talks concerning stages of both low-mass and high-mass star formation were given, as was a theoretical talk on a new class of models that combines hydrodynamics with chemical simulations. The first session of hot results from Herschel emphasized observations of water vapor, molecules in protostellar shocks, and a wide spectral survey toward Orion KL.

Astrochemistry certainly extends to planetary studies. This session started with a review talk on the chemistry of the solar system, including the origin of water on Earth, which was followed by talks on comets, meteorites, and the atmospheres of Titan and Saturn. The power of sample return missions to solar system bodies was emphasized.

The second day started with a session on evolved stars, in which supernova chemistry was also discussed. Talks on the molecular evolution from AGB stars to planetary nebulae, the role of time-dependent anionic chemistry (involving negatively-charge molecules) in IRC+10216, and the detection of fullerenes in assorted environments rounded out the session. Complex molecules were subsequently discussed in a variety of objects, along with current gas-grain simulations as well as possible future simulations involving the use of stochastic methods to improve the surface chemistry occurring in icy mantles.

Astrochemistry is based on the laboratory and theoretical study of basic atomic and molecular processes, and two sessions were held on this subject. The first concerned gas-phase processes, where a review talk was given on gas-phase reactions as a function of temperature, followed by a talk concerning the theory of low-temperature reactions, and one on experimental studies on the rates of reactions involving anions and how they relate to the observations of such species in various sources. Surface processes in the laboratory and in space were discussed in the second session (on day 3). Much progress has been made during the last decade, but there is still a great need for further laboratory studies before robust interstellar chemical simulations including surface processes can 
be constructed. The second day of the meeting ended with another Herschel hot topic session, highlighted by the report of an unambiguous detection of interstellar $\mathrm{O}_{2}$.

The topic of protoplanetary disks on day three demonstrated vividly the phenomenal developments in observations at a variety of wavelengths ranging from the millimeter to the far-UV and an emphasis on interferometry. Modeling was also discussed, as was the chemical history of molecules from the hot core to the disk stage.

Although most of astrochemistry still revolves around galactic sources, the field of extragalactic astrochemistry will receive a big boost with the onset of ALMA observations. So, it was quite appropriate to have a session on extragalactic astrochemistry, which was held on day 4 , including talks on the early universe chemistry and on extragalactic line surveys. It is impressive to now see spectra of extragalactic sources with similar complexity to those found in galactic star-forming regions three decades ago!

Next in line was the explosive topic of exoplanets and their atmospheres. Talks on observations, atmospheric models and their chemistry, as well as biomarkers of habitable worlds were included. The final session on day 4 concerned the tools of analysis and databases, including how to reduce the problem of unidentified lines in hot cores, on various analysis tools for spectral surveys, and on database uses.

The last day started with a session on diffuse clouds and photon-dominated regions (PDRs). The role of turbulence in diffuse clouds was discussed. Overview talks on both PDRs and XDRs as well as diffuse interstellar bands were given. A number of aspects of the PAH hypothesis were touched upon. Finally, the complex nature of the central molecular zone of our galaxy, as seen through $\mathrm{H}_{3}^{+}$, was explored. Next came the third of the Herschel sessions, which included talks on observations of diffuse clouds in the spiral arms of the Milky Way, and carbon chemistry in translucent clouds. The detection of the reactive ions $\mathrm{OH}^{+}$and $\mathrm{H}_{2} \mathrm{O}^{+}$in a variety of sources was an exceptionally interesting topic. The oral program was concluded with a thoughtful summary of the field, past, present, and future, by John Black.

\section{Closing remarks}

Based on the growth of the field, the sustained activity of the working group for over 25 years, and exciting future prospects, the WG proposes to elevate Astrochemistry to a proper commission under Division VI (new Division H). Among the activities that can be enhanced as a full-fledged commission are broader advertising of the role of astrochemistry and its multiple uses in astronomy; emphasizing the large amount of new spectra coming from new telescopes and the need for improved molecular data; expanding the training of astrochemistry among astronomers; and stimulating further interdisciplinary activities. By the time of the next astrochemical symposium, planned for 2017, much progress will have been made thanks to Herschel and ALMA, and the field will have grown both in size and, we trust, in understanding.

$$
\begin{array}{r}
\text { Ewine F. van Dishoeck } \\
\text { chair of Working Group } \\
\text { Eric Herbst } \\
\text { vice-chair of Working Group }
\end{array}
$$

\section{Reference}

Cernicharo, J. \& Bachiller, R. 2012, The Molecular Universe, IAu Symposium 280, Toledo, Spain, 30 May - 3 June 2011, CUP, in press 